\title{
Incorrect Booklet Given
}

National Cancer Institute

\section{Source}

National Cancer Institute. Incorrect Booklet Given. NCI Thesaurus. Code C106473.

The wrong booklet was provided to an individual. 